\title{
FLITECAM: current status and results from observatory verification flights
}

Ian S. McLean, Erin C. Smith, E. E. Becklin, E. W. Dunham, Jennifer W. Milburn, et al.

Ian S. McLean, Erin C. Smith, E. E. Becklin, E. W. Dunham, Jennifer W. Milburn, Maureen L. Savage, "FLITECAM: current status and results from observatory verification flights," Proc. SPIE 8446, Ground-based and Airborne Instrumentation for Astronomy IV, 844619 (24 September 2012); doi: $10.1117 / 12.926821$

Event: SPIE Astronomical Telescopes + Instrumentation, 2012, Amsterdam, Netherlands 


\title{
FLITECAM: current status and results from observatory verification flights
}

\author{
Ian S. McLean*a , Erin C. Smith ${ }^{\mathrm{b}}$, E. E. Becklin ${ }^{\mathrm{c}}$, E. W. Dunham ${ }^{\mathrm{d}}$, Jennifer W. Milburn ${ }^{\mathrm{e}}$, and \\ Maureen L. Savage ${ }^{c}$ \\ ${ }^{a}$ University of California, Los Angeles, CA, USA 90095-1547; \\ bNASA Ames Research Center, Moffett Field, CA, USA 94035; \\ ${ }^{c}$ USRA, NASA Ames Research Center, Moffett Field, CA, USA 94035 \\ ${ }^{\mathrm{d}}$ Lowell Observatory, Flagstaff, AZ, USA 86001; \\ ${ }^{\mathrm{e}}$ California Institute of Technology, Pasadena, CA, USA 91125
}

\begin{abstract}
This paper describes the current status of FLITECAM, the near-infrared $(1-5 \mu \mathrm{m})$ camera and spectrometer for NASA's Stratospheric Observatory for Infrared Astronomy (SOFIA). Due to a change in schedule FLITECAM's delivery was advanced, allowing it to be co-mounted with the HIPO instrument and used on four flights in October 2011 for observatory verification. Although not part of FLITECAM's commissioning time, some preliminary performance characteristics were determined. Image size as a function of wavelength was measured prior to the installation of active mass dampers on the telescope. Preliminary grism spectroscopy was also obtained. In addition, FLITECAM was used to measure the emissivity of the telescope and warm optics in the co-mounted configuration. New narrow band filters were added to the instrument, including a Paschen alpha filter for line emission. Results are illustrated.
\end{abstract}

Keywords: spectrometer, near-infrared, SOFIA, airborne astronomy

\section{INTRODUCTION}

FLITECAM, the near-infrared $(1-5 \mu \mathrm{m})$ camera and spectrometer for SOFIA, has been described in several earlier papers ${ }^{1,2}$. Briefly, FLITECAM has a circular field of view of $\sim 8^{\prime}$ diameter inscribed within a $1024 \times 1024$ InSb Aladdin III detector. There are two primary observing modes, direct imaging and grism spectroscopy using fixed slits. In addition, there is a special rapid-exposure "movie" mode for occultation studies, as well as a pupil-viewing mode intended primarily for observations near 3.0 - 3.5 microns. By designing FLITECAM to operate in a vertical orientation at the Cassegrain focus of the Lick Observatory Shane telescope $(3.0 \mathrm{~m}, \mathrm{f} / 17)$ as well as in the horizontal orientation at the Nasmyth focus of SOFIA $(2.5 \mathrm{~m}, \mathrm{f} / 19.7)$, the instrument could be tested and evaluated independently of the SOFIA platform, except for the very longest wavelengths where ground-based backgrounds are too high. FLITECAM was deployed to Lick Observatory eight times prior to installation on SOFIA. Observations made during the runs at Lick Observatory resulted in three scientific papers ${ }^{3,4,5}$, several conference papers and two successful Ph.D. theses (Amanda K. Mainzer and Erin C. Smith).

During the summer and fall of 2011, FLITECAM was delivered to SOFIA and co-mounted with HIPO, the High-speed Imaging Photometer for Occultations (PI Edward Dunham, Lowell Observatory). HIPO is mounted on-axis and FLITECAM goes above it. A periscope composed of a dichroic beam-splitter and fold-mirror in front of HIPO reflects infrared light to FLITECAM. This instrument combination (FLIPO) was used during line operations (line ops) with the plane on the ground and four observatory verification flights in October 2011. These were not instrument commissioning flights because the Observatory itself was still under development. However, useful information was gained on hardware and software performance. In this paper we present a brief update of FLITECAM's properties and discuss the results from those early non-commissioning flights.

*mclean@astro.ucla.edu

Ground-based and Airborne Instrumentation for Astronomy IV, edited by lan S. McLean, Suzanne K. Ramsay, Hideki Takami, Proc. of SPIE Vol. 8446, 844619 - ( ) 2012 SPIE · CCC code: 0277-786X/12/\$18 - doi: 10.1117/12.926821 


\section{INSTRUMENT STATUS AND UPDATE}

FLITECAM can be configured as a wide-field camera with a suite of both narrow-band and broad-band filters, or as a long-slit spectrometer using slits of fixed width and height plus one of three grisms. For the 1" slit the resolving power is $\mathrm{R} \sim 2,000$. In imaging mode the pixels subtend $0.475^{\prime \prime}$ on SOFIA. Image quality is very good over the inner $5.6^{\prime}$ diameter field where it will be limited by turbulence; images at the edge of the full $8^{\prime}$ field are limited by residual coma in the optical design, which was originally intended for a 512x512 array. To implement the spectroscopic mode a set of three direct-ruled KRS-5 grisms ${ }^{6,7}$ are used, together with a suite of custom order-sorting filters and a pair of fixed slits in a deployable focal plane mask. The detector layout for these two modes is illustrated in Figure 1. Because the detector has bad corners, and to get the best image quality, only the inner 5.6 x 5.6' area is extracted from dithered observations.

A third mode, which is a special case of the imaging configuration, allows rapid acquisition of frames or sub-frames for occultation studies. Yet, another special case of the imaging configuration is the Pupil-Viewing (PV) mode, in which additional lenses are moved into the beam to re-image the entrance pupil onto the detector. This mode is designed to work mainly from $3.0-3.5 \mu \mathrm{m}$ in order to evaluate the emissivity of SOFIA's primary mirror.

\section{InSb Detector Format: 1024 x 1024 pixels Pixel size on sky: $0.475^{\prime \prime}$ x 0.475"}
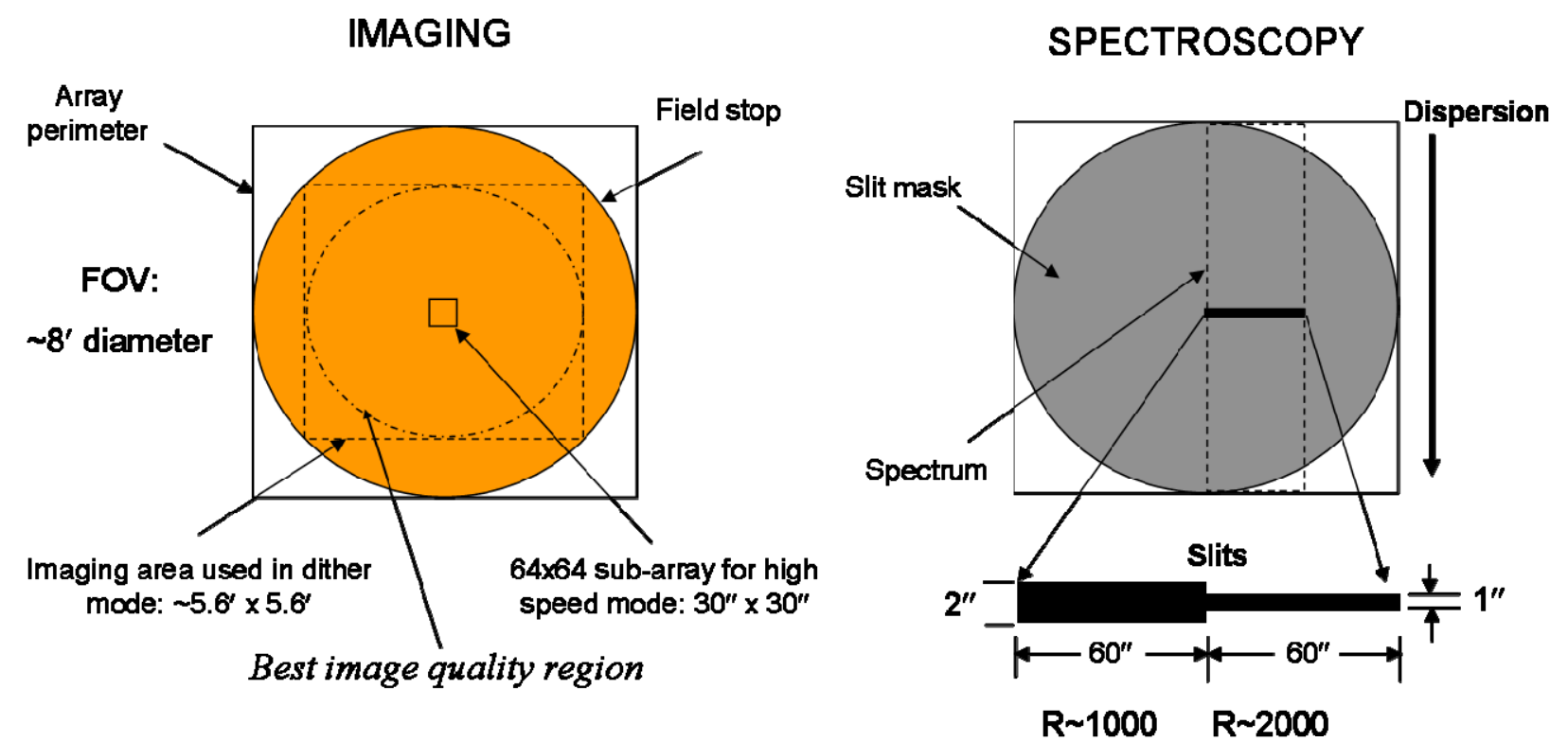

Figure 1. The detector layout for FLITECAM's imaging and spectroscopic modes is shown. A slit mask mechanism in the cold focal plane is used to switch between modes. Three grisms are located in the rear filter wheel. Each can be used in three orders by selecting an appropriate filter in the front wheel.

To avoid condensation on the large entrance window (140 $\mathrm{mm}$ clear aperture), which cools by radiation to the cold internal surfaces, a flow of dry nitrogen across the window is maintained for ground-based use. On board SOFIA, the cavity in front of the window can be sealed and evacuated for take-off and landing. However, a dry nitrogen purge is still required for "line operations" or ground-based testing with SOFIA, and for laboratory work involving the telescope simulator. For co-mounted operation with HIPO a modified flow line was designed and installed. The vacuum window is $21.5 \mathrm{~mm}$ thick to ensure negligible distortion due to the pressure differential. The beam is collimated and folded through a dual filter wheel, then folded back through a camera assembly onto the InSb detector. The InSb detector is controlled by a custom set of electronics made by Mauna Kea Infrared (MKIR, HI). Electronics mounted on the cryostat read out the detector, amplify and convert the signals to digital packets, and transmit them via fiber optic cable to the MKIR computer located in a remote rack called the "PI" rack. Astronomers can adjust the exposure time and the readout mode of the detector through the astronomer's graphical user interface on the host computer in the PI rack. The fastest 
exposure time for the full 1024 x 1024 array is 0.3 seconds. A sub-array of 512 x 512 pixels can be read out in 0.1 seconds and a 64 x 64 sub-array allows 0.02 second exposures $(50 \mathrm{~Hz}$ frame rate). FLITECAM has four cryogenic mechanisms: an aperture slide, which moves the slit mask in and out of the beam for spectroscopy, two independent filter wheels, and another slide which carries the pupil-viewing doublet in and out of the beam immediately in front of the $f / 5$ camera. Stepper motors prepared for cryogenic operation inside the instrument are used together with a series of microswitches for positioning each of these mechanisms. The cryostat is a thick-walled cylinder about $1.52 \mathrm{~m}(5 \mathrm{ft})$ long and $0.49 \mathrm{~m}(1.6 \mathrm{ft})$ in diameter. The front half of the cryostat houses the opto-mechanical system and the InSb detector, while the back section holds a pair of 20 liter liquid cryogen reservoirs, one for liquid nitrogen and the other for liquid helium. Figure 2 shows FLITECAM co-mounted with HIPO on the airplane in October 2011.

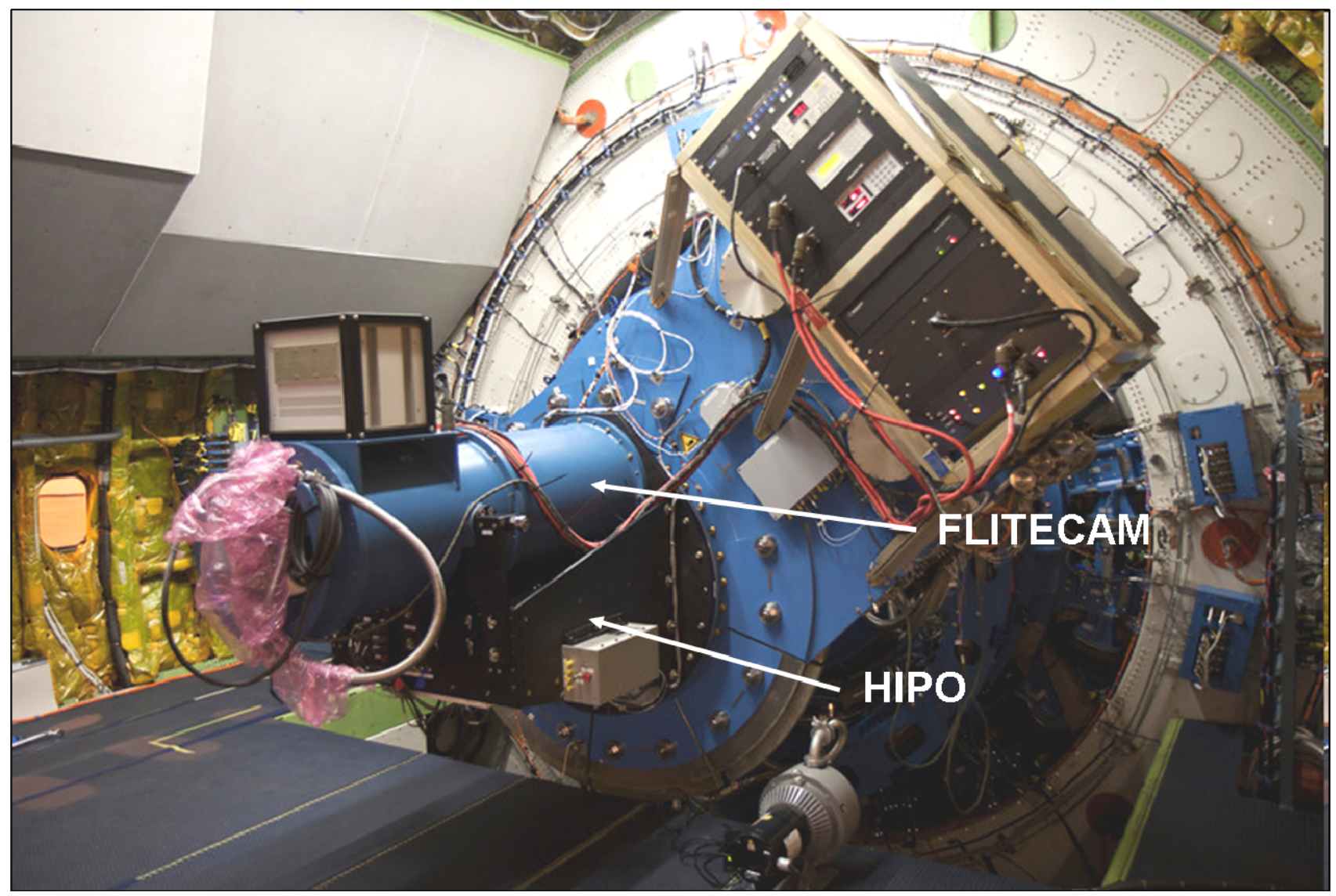

Figure 2. FLITECAM (blue cylinder) co-mounted with HIPO (black) on SOFIA. The FLITECAM counterweight rack electronics is upper right. The telescope is at its nominal elevation of 45 degrees.

Prior to the SOFIA test flights in October 2011, the FLITECAM filter set was modified from those used during the Lick Observatory deployments. Six new narrow-band filters were procured from Materion Barr Precision Optics and Thin Film Coatings (formerly Barr Associates). The complete filter set now consists of six standard broad-band filters, four custom order-sorting filters and a set of six narrow bands. Broadband imaging filters are available in the standard J, H, $\mathrm{K}, \mathrm{L}, \mathrm{L}$ ', and $\mathrm{M}$ passbands. Order sorting filters (OSFs) for use with the grisms in spectroscopic observations are designated $\mathrm{H}_{\text {wide }}, \mathrm{K}_{\text {wide }}, \mathrm{K}_{\text {long }}$ and $\mathrm{L}+\mathrm{M}$. The new narrow-band imaging filters are $1 \%$ filters for Paschen $\alpha(1.875 \mu \mathrm{m})$ and Paschen $\alpha$ continuum $(1.900 \mu \mathrm{m})$, and $4 \%$ filters for PAH $(3.30 \mu \mathrm{m})$, Ice $(3.05 \mu \mathrm{m}), \mathrm{L}_{\text {narrow }}(3.61 \mu \mathrm{m})$, and $\mathrm{M}_{\text {narrow }}$ $(4.81 \mu \mathrm{m})$. Grism spectroscopy is performed with three different grisms, each of which can operate in 3 orders, yielding nearly contiguous coverage from $1-5.5 \mu \mathrm{m}$. The only gap occurs at the $4.2-4.4 \mu \mathrm{m}$ atmospheric band. Figure 3 shows the FLITECAM filter pass bands overlaid on an atmospheric transmission model based upon the ATRAN code assuming a zenith angle of 45 degrees at an altitude of $41,000 \mathrm{ft}$, with an $\mathrm{H}_{2} \mathrm{O}$ burden of $7 \mu \mathrm{m}$. 


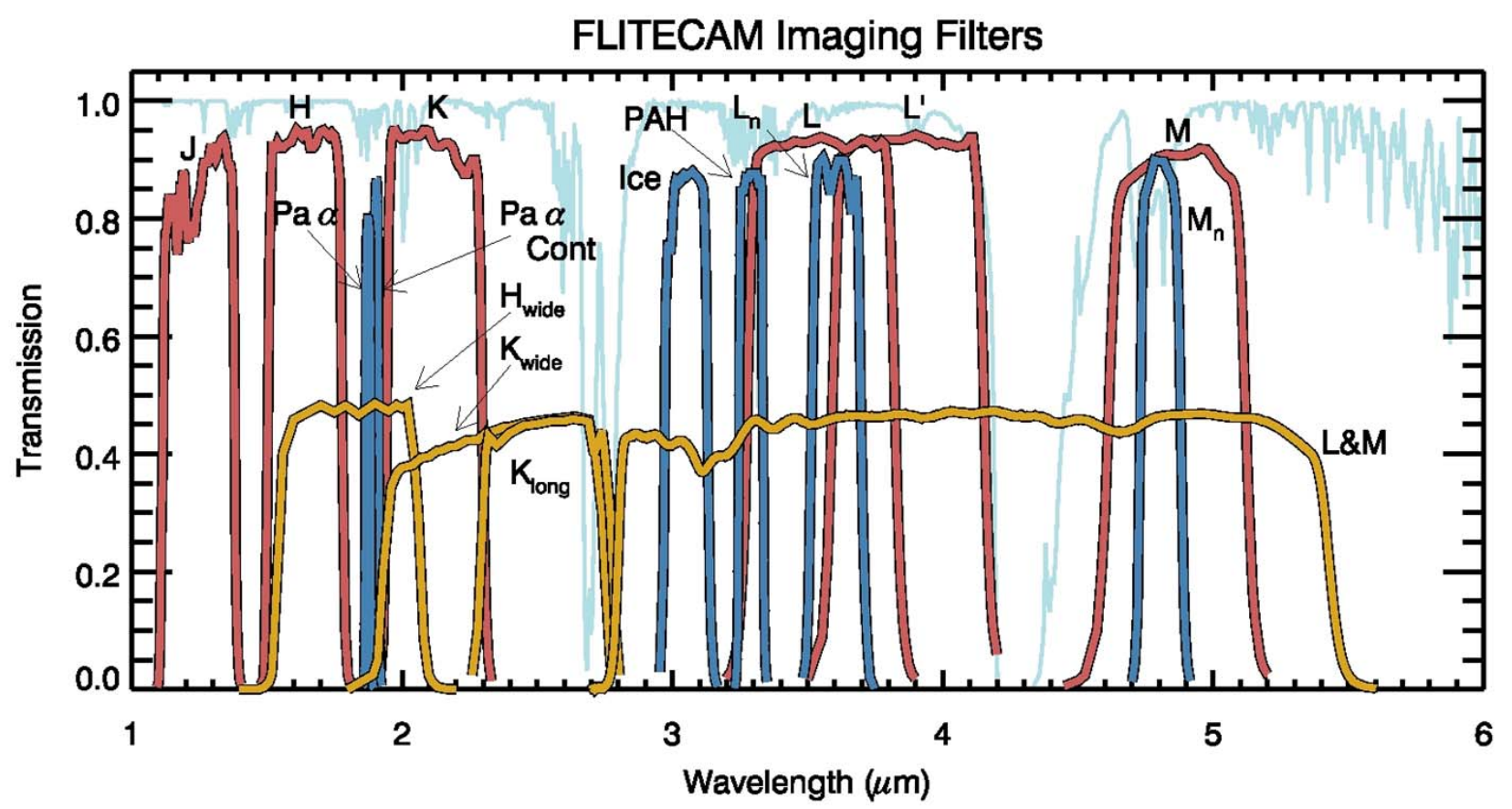

Figure 3. This plot shows the FLITECAM filter pass bands overlaid on an atmospheric transmission model.

After installation of the narrow-band filters it was noticed that thermal backgrounds inside the instrument were higher than previously observed, perhaps due to light leakage in the dual filter wheel. No changes were made prior to first flights. After delivery to the Dryden Aircraft Operations Facility (DAOF) in Palmdale, California, the electronics systems in both the counterweight rack on the telescope and the PI/computer rack were over-heated accidentally when the aircraft was washed outside the hangar on an extremely hot day with the cabin sealed and not air-conditioned. Several system components were damaged including a Lakeshore temperature controller and a DSP board in the MKIR electronics. The Lakeshore unit was replaced quickly with a spare, but as a result of the DSP fault one quadrant of the detector array could not be read out properly. The observatory verification flights were carried out with these impediments. The DSP board has since been repaired and all quadrants of the detector are reading out again.

\section{RESULTS FROM OBSERVATORY COMMISSIONING FLIGHTS}

\subsection{Pre-flight tests and preparations}

Prior to installation on the aircraft at the DAOF, HIPO and FLITECAM were co-mounted on a test structure, the TAAS (Telescope Assembly Alignment Simulator) which simulates the mechanical interface of the SOFIA telescope mounting flange and also provides an optical beam for alignment and bore-sight evaluation. A series of tests using LN2-cooled targets on the TAAS gave information about the emissivity of the FLITECAM window ( $9 \%)$ and the warm periscope optics for the FLIPO configuration $(\sim 11 \%)$ for a total of $\sim 20 \%$. Measurements were also made of the relative pixel positions of optical targets in the two imaging systems. Handling and safety procedures were evaluated and developed using the TAAS.

One night of line ops was carried out successfully on October 11. It was challenging to keep the FLITECAM window dry, either by a regulated flow of dry N2 gas or by closing the gate valve and pumping on the tub. Thermal images and visual inspection reveal that there are already some small water spots near the center of the CaF2 window. The flight series occurred on the nights of October 13, 17, 20 and 24. The first two flights were from DAOF to NASA Ames and then Ames back to DAOF after a weekend lay-over. For the second pair of flights the plane took off from and returned to the DAOF in Palmdale. The FLITECAM team comprised Ian McLean, Erin Smith, Eric Becklin and Maureen Savage for all flights. George Brims (UCLA) joined for the first flight in case of technical problems and Jennifer Milburn (CIT) for the first two flights to support software testing and evaluation. 


\subsection{In-flight results}

FLITECAM was operated successfully in the FLIPO configuration on four flights in October 2011. While observatory characterization was a major component of these flights, a significant portion of time was reserved for HIPO and FLITECAM characterization. The main purpose of these characterization activities was to support SOFIA's Cycle 1 observing call, rather than to formally commission FLITECAM. Formal commissioning will occur in 2013.

Using standard stars, the throughput and zeropoints were derived. For $\mathrm{J}, \mathrm{H}$ and $\mathrm{K}$ the results were as expected from ground-based measurements. Sensitivity in the thermal IR was reduced in the FLIPO configuration due to the presence of additional warm optics in the beam and a probable light leak in the filter wheel. Pupil-viewing and out-of-focus images indicate good alignment of FLITECAM to the telescope axis and secondary mirror. Emissivity and/or thermal background were higher than expected. Lab tests already indicated that, in the FLIPO configuration, FLITECAM sees an emissivity of $20 \%$. Adding the telescope pushes this up to about $35 \%$. Images could not be obtained in broad band M, even with a small sub-array. Image quality varied from 9 to 6 pixels, or 4.5 to 3 seconds of arc, from J (1.25 microns) to narrow band $\mathrm{L}$ at 3.6 microns. Figure 4 illustrates two of the first light images, a standard star and the Orion Nebula. A one hour test of photometric stability on a bright star gave standard deviation errors $\sim 0.007$ magnitudes.
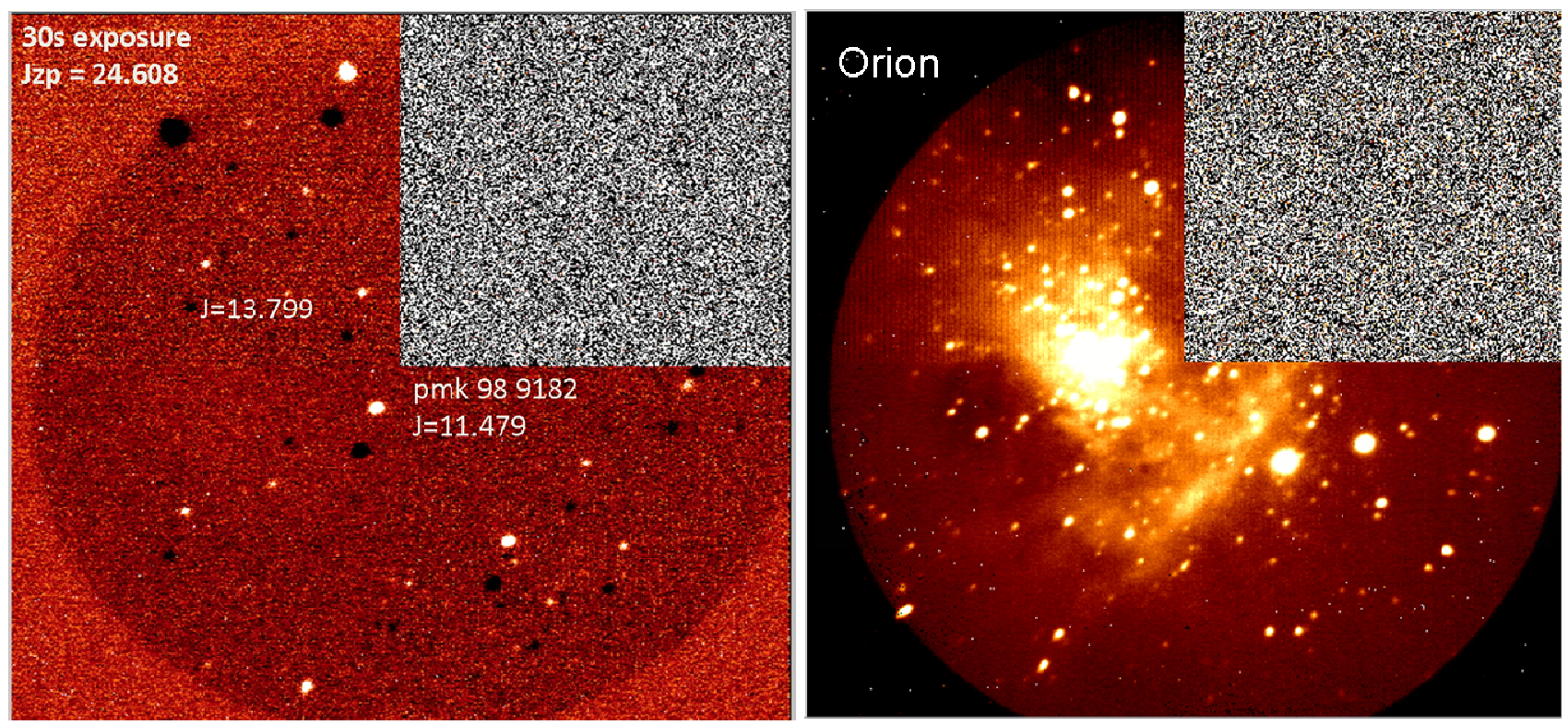

Figure 4. First light images. The missing quadrant is evident. Image quality was limited by turbulence and by crosselevation telescope shake. The image on the left is J-band and that on the right is K-band.

Spectroscopy was obtained using several grism settings from 2-5.5 microns. The PAH feature at 3.3 microns was seen in many sources and water lines that are saturated for ground-based observations were not for SOFIA. It was extremely difficult to place a star at a desired location on our slit, either the 1" or the 2" wide slits. Small telescope moves to tweak the position often failed to move as predicted. Nevertheless, many spectra were obtained, both as ABBA and on/off nods. Some results from the October flights are presented in Figure 5

Spectra from 3-5 microns were taken as a function of telescope elevation angle to look for scattered thermal continuum emission from the outboard engine's hot tail cone as well as possible line emission from the exhaust plume. There was a definite increase in signal by $\sim 20 \%$ at lower elevations but no spectral features were detected. This is consistent with the original predictions for the SOFIA engine plume ${ }^{8}$ Images at 3.0-3.5 microns using the pupil-viewing mode were also obtained as another way to look for excess background as a function of telescope elevation. Again there was an increase in signal at lower elevations. In imaging mode the signal was mainly a flare of emission which, unfortunately, was mostly in the missing quadrant. Again this excess is at least qualitatively consistent with that predicted by engine plume simulations and analysis. ${ }^{8}$ HIPO results and general results from all the SCAI flights are reported elsewhere. ${ }^{9,10}$ 

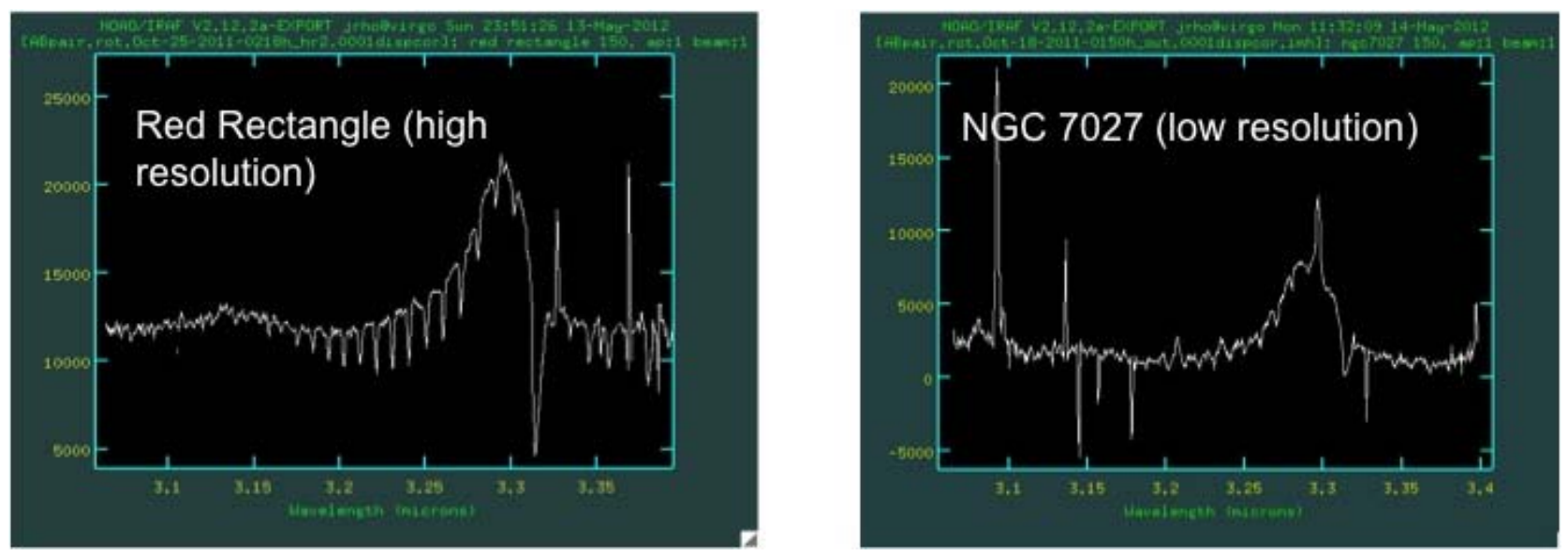

Figure 5. FLITECAM spectra at 3 microns obtained in high (left) and low (right) resolution modes. Emission from Polycyclic Aromatic Hydrocarbons (PAH) is shown at 3.3 microns.

Interactions between FLITECAM and the telescope control software (MCCS) were highly variable. There was some evidence of a difference in behavior depending on which guide camera was used in observations. For these flights, FLITECAM performed its dither and slit-nodding moves by sending absolute coordinates. Dither patterns and nods performed while tracking with the Focal Plane Imager (FPI) was measurably more effective than when tracking with the Fine Field Imager (FFI). This behavior needs more testing on the Hardware In the Loop Simulator (HILS) and on during line ops. Based in part on the experience gained during October 2011, the interface to the SOFIA telescope control system is currently undergoing a major revision that will be available for the next flight series. FLITECAM will now take advantage of the newly available dither and nod commands supported by the telescope control system to eliminate the effects of telescope position reporting errors.

Attempts to test the real-time data reduction pipeline for imaging were not very effective due to the presence of the bad quadrant. Off-line reductions were more successful. We obtained good images of W3, Orion, NGC7027 and the Red Rectangle using the Paschen-alpha filter and the 3.3 micron PAH filter. These initial results are encouraging. Figure 6 illustrates reduced images for Orion and W3.
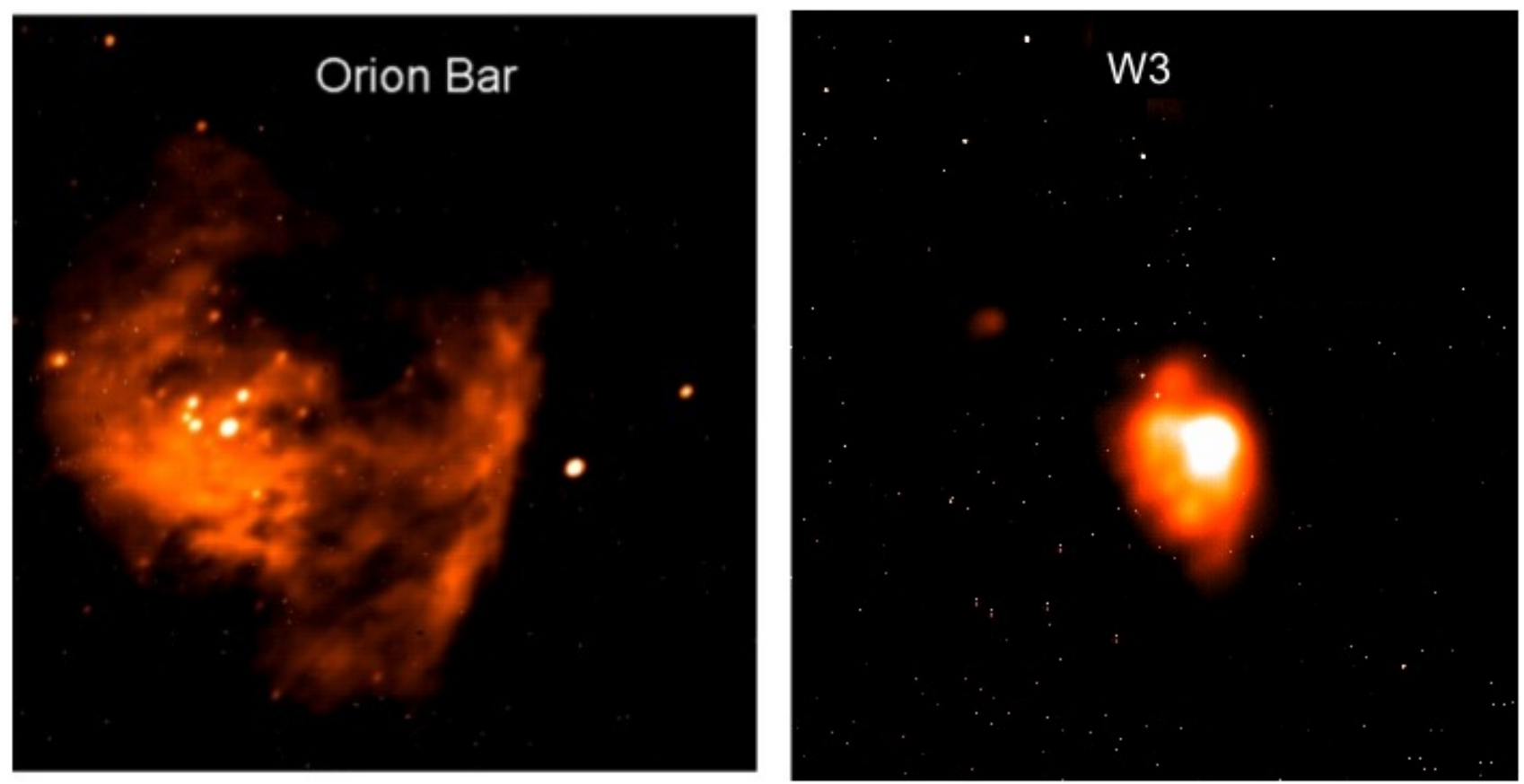

Figure 6. Reduced images from dither patterns on the Orion Bar and W3 using the 1\% Paschen-alpha filter at $1.875 \mu \mathrm{m}$. 


\section{CONCLUSIONS}

When FLITECAM was tested at Lick Observatory the telescope control interface was developed to use simple RA and Dec offsets. Unfortunately, simple offset moves in RA and Dec were not supported by the SOFIA telescope control system during the October 2011 flight series. Instead, the FLITECAM code was modified to use instrument coordinates (i.e. pixel coordinates) for all telescope moves except for dither pattern moves. Dither patterns were performed by polling the telescope position and then calculating the coordinates of the next dither position in RA and Dec. Latency problems in the system would sometimes result in erroneous telescope position being reported by the telescope control system which then caused errors in the calculated RA and Dec for the next dither position. FLITECAM's user interface has been updated to the newest Java JDK (1.7) and all dependent packages (e.g. JSky) are the most current versions available. Currently, the interface to the SOFIA telescope control system is also undergoing a major revision. FLITECAM will now take advantage of the newly available dither and nod commands which will eliminate the problem of telescope position reporting errors. Another lesson learned during line operations and initial flights was the importance of associating tracking information with any and all telescope moves. Every telescope move must be associated with the currently active AOI for tracking the telescope position. Failure to associate a move with the currently active AOI results in the telescope move being reversed by the tracking system.

Imaging was carried out in most filters using both full-array and sub-array readouts, and several 5-point dither patterns were executed. Spectroscopic tests used both the low and high resolution slits and most grism/filter combinations were exercised. Among the tests accomplished were gyro drift observations, emissivity tests, a check for emission from engine exhaust, image size as a function of wavelength, and of course throughput and backgrounds.

Despite a missing quadrant, excess emissivity and anomalous thermal backgrounds, this initial co-mounted test of FLITECAM and HIPO was successful and preliminary results were encouraging. Sensitivity is as expected in J, H and K but we can't confirm L and M yet. Photometric stability is unknown at levels $<0.007$ magnitudes. Image quality appears to improve towards longer wavelengths reaching a minimum around 3 microns. The narrow (1\%) Paschen-alpha filter at 1.88 microns and the adjacent continuum filter at 1.90 microns are very effective despite being in a region very sensitive to water vapor. Clear evidence of image jitter in the cross-elevation direction was observed and thermal emission due to engine exhaust was evident for elevations $<35^{\circ}$.

The interaction among the instruments, telescope control software and the guider systems clearly requires more development. Within FLITECAM, there is evidence of glints and light leaks when using the new narrow-band filters unless they are "blocked" with one of the broad order-sorting filters used for spectroscopy. This behavior was seen in the lab prior to flights but it was hoped that having a well-defined telescope beam would reduce the effect. FLITECAM will be refurbished before the next flights.

\section{ACKNOWLEDGEMENTS}

The authors wish to acknowledge the hard work of the entire FLITECAM project team, past and present, including Ted Aliado, George Brims, John Canfield, Don Figer, John Goulter, Jochen Horn, Evan Kress, Ken Magnone, Nick Magnone, Amy Mainzer, Andrew Oldag, Mike Spencer, Gunnar Skulason and Tatyana Silvers. We are also grateful to Bill Vacca and Jeonghee Rho, and all the SOFIA staff, for support in-flight and with data analysis. FLITECAM is primarily funded by a grant from USRA.

\section{REFERENCES}

[1] McLean, I. S.; Smith, E. C.; Aliado, T.; Brims, G.; Kress, E.; Magnone, K.; Milburn, J.; Oldag, A.; Silvers, T.; and Skulason, G., "FLITECAM, a 1-5 micron camera and spectrometer for SOFIA," Proc. SPIE, 6269, 195-204 (2006).

[2] McLean, I. S. and Smith, E. C., "Ground-based commissioning of FLITECAM," Proc. SPIE, 7014, 34-42 (2008).

[3] Mainzer, A. K. and McLean, I. S., "Using narrowband photometry to detect young brown dwarfs in IC 348," APJ, 597, 555-565 (2003).

[4] Mainzer, A. K.; McLean, I. S.; Sievers, J. L. and Young E. T., "Using narrowband photometry to classify stars and brown dwarfs," APJ, 604, 832-841 (2004). 
[5] Smith, E. C. D. and McLean, I. S., “A survey of 3.3 micron PAH emission in Planetary Nebulae”, APJ, 676, 408415 (2008).

[6] McLean, I. S., [Electronic Imaging in Astronomy: detectors and instrumentation], Springer, Berlin Heidelberg New York, 176 (2008).

[7] Smith, E. C. and McLean, I. S., "Grism spectroscopy with FLITECAM,” Proc. SPIE, 6269, 56-63 (2006).

[8] Dinger, A.S., Dunham, E. W., Erickson, E. F., Klotz, S., Koch, D. and Yee, R. "Evaluation of Jet-Exhaust Emission Scattering for the Aft Telescope Configuration on SOFIA," Ames Research Center NASA Technical Memo, 110828, 1-22 (1992).

[9] Dunham, E. W., Bida, T. A., Collins, P. L., Mandushev, G. I., McLean, I. S., Person, M. J., Smith, E. C., Taylor, B. W. and Zoonematkermani, S., "HIPO in-flight performance aboard SOFIA," Proc. SPIE, 8446-42, this conference, (2012).

[10] Temi, P., et al., "SOFIA observatory performance and characterization," Proc. SPIE, 8444-39, this conference, (2012). 